\title{
Talilmogene laherparepvec generates systemic T-cell-mediated anti-tumor immunity
}

\author{
Julia Piasecki, Le Tiep, Jean Zhou, Courtney Beers \\ From Society for Immunotherapy of Cancer 28th Annual Meeting \\ National Harbor, MD, USA. 8-10 November 2013
}

Talimogene laherparepvec is an investigational oncolytic immunotherapy based on a modified herpes simplex virus type-1 (HSV-1) that is designed to selectively replicate in tumor tissue and to stimulate a systemic antitumor immune response. Intralesional administration of talimogene laherparepvec is intended to result in oncolysis within injected tumors. Iterative viral replication of virus within permissive tumor tissue results in lytic cell destruction and local release of progeny virus and cellular derived antigens. GM-CSF, a product of the viral transgene, is also produced locally such that it can recruit and stimulate antigen presenting cells which, in addition to relevant tumor-derived antigens, are required for the initiation of a systemic antitumor immune response. A phase 3 clinical trial of talimogene laherparepvec in regionally or distantly metastatic melanoma has recently reported a $26 \%$ ORR objective response rate (6\% for GM-CSF alone) $\{(\mathrm{J}$ Clin Oncol 31, 2013 (suppl; abstr LBA9008)\}. Since the immune mediated anti-tumor mechanisms have yet to be fully understood, we sought to identify immune-specific changes post talimogene laherparepvec administration in the A20 syngeneic contralateral tumor model. We analyzed immune populations in both injected and noninjected tumors and in peripheral hematopoietic tissues at 2, 5 and 10 days post injection. Animals received one $5 \times 10^{6}$ PFU intratumoral dose of talimogene laherparepvec which induces complete regression in approximately $70-100 \%$ of the injected tumors and approximately $50-60 \%$ of the contralateral non-injected tumors. Injection of virus resulted in marked increases in injected tumor draining lymph node and spleen size compared to control animals. Upon further flow cytometric analysis, changes in T-cell populations were observed in the injected and noninjected tumor draining lymph nodes, spleen and peripheral blood and T-cells expressed higher levels of cell

Amgen, Seattle, WA, USA 